\title{
Manipulation for cervical spinal dislocation under general anaesthesia: serial review for 4 years
}

\author{
XH Xiong, A Bean, A Anthony, G Inglis and D Walton \\ Christchurch Spinal Injuries Unit, Burwood Hospital, Christchurch, New Zealand
}

\begin{abstract}
Manipulation under anaesthesia is an important method to reduce cervical spinal dislocations in the acute stage. Causes of failure have not been clearly identified and neurological complications can be the major concern. All cervical dislocations have been traditionally treated by manipulation under anaesthesia in the Christchurch Spinal Injuries Unit as the primary treatment. We reviewed all 31 patients treated from 1991-1995, with detailed documentation of neurological progression and final outcome. Three patterns were identified: bilateral dislocation, unifacet dislocation and fracture dislocation. Most of the dislocations (74\%) were successfully reduced by manipulation alone with minimum complications. The remaining $26 \%$ patients required open reduction. The predominant causes of failure of reduction by manipulation were co-existing fractures. The success rate of reduction by manipulation was $90 \%$ for pure bifacet and unifacet dislocations, but was only $22 \%$ for the fracture dislocations. The study concluded that manipulation under anaesthesia is a safe and effective procedure for pure cervical spinal dislocations. Fractures related to the dislocation should be identified early and open reduction be considered.
\end{abstract}

Keywords: dislocation; reduction; cervical spine; manipulation under general anaesthesia

\section{Introduction}

Cervical spinal dislocations require urgent management with great experience and skill. Yet no unified recommendations have been accepted universally among the three approaches: closed manipulation (MUA), increasing skull traction and open reduction. ${ }^{1,2,5}$ There have been increasing concerns over the safety of closed reduction which may cause neurological deterioration particularly secondary to herniated discs. ${ }^{1-4}$ Recent studies however indicated that MRI might be over sensitive as there was much less reported neurological involvement related to disc lesions even though the latter were found in up to $80 \%$ of dislocations. $1,5,10$

Manipulation under anaesthesia is an effective way of reducing the dislocations. However, the failure rate can be high (26\% in our series and $27 \%$ in Lee's series 1994). Our study is aimed at explaining why some dislocations could not be reduced by manipulation. This study also presents the detailed outcomes of all patients who showed favorable recovery and reflect the quality of current spinal rehabilitation. The safety and success of the manipulative reduction of cervical dislocation will be discussed.

\section{Patients and methods}

Thirty-one patients with traumatic cervical dislocations have been included in the study. There were three

Correspondence: XH Xiong patterns of dislocation: (1) bifacet dislocation without fractures; (2) unifacet dislocation without fractures; (3) fracture dislocation involving either one or both facets. The causes of injury included Motor Vehicle Accidents (MVA) in 15 cases and Rugby injuries in 11 cases. The patients were young adults $(20-40$ years $)$ in $66 \%$ of cases (14 were between 20 - 30 years and seven between $31-40$ years), but age ranged from 16 to 79 years old. Twenty-four of the patients were male and seven female.

Pre-reduction investigations included A-P and lateral plain X-ray for all the patients and tomography, oblique views of CT scans for selective cases, particularly when fracture(s) were suggested by plain films.

The manipulation is a carefully controlled procedure involving total relaxation with general anaesthesia and muscle relaxants, gentle traction by the operator holding the chin and occiput and counter traction by the assistant holding the wrists from the foot of the bed. A 'click' will be felt, especially by the operator on reduction. An X-ray image intensifier is required to confirm the reduction. Patients with nonreducible dislocations remain in traction with somewhat improved alignment to be further investigated by $\mathrm{CT}$ and/or MRI. Alternatively, open reduction will be performed.

\section{Results}

All unifacet dislocations and bifacet dislocations except one, were successfully reduced by manipulation alone 
(Table 1). Of the patients with fracture dislocation however, seven out of nine attempts were unsuccessful and required open reduction and internal fixation (Table 1).

All patients were internally fixed by ' $\mathrm{H}$ ' plate fixation via an anterior approach once reduction was achieved. Patients with un-reduced dislocations were treated by open reduction and wiring fixation posteriorly (Table 2).

Non-reducible dislocations were related to coexisting fractures in seven out of eight patients. The most common fractures were lateral mass fractures with facet involvement (in seven of the 11 patients). Other patterns identified included fractures of the vertebral body (in three cases) and transverse process (in one case). The timing of reduction or attempt of reduction under general anaesthesia were $4-27 \mathrm{~h}$ post accident with an average of $13.6 \mathrm{~h}$ in this series. The neurological impairments were classified into four groups: complete; incomplete; minimal (with root involvement, segmental or temporary impairment) and none (Table 3).

Neurological outcomes were assessed according to Frankel scale. All patients with complete lesions remained complete, while patients with incomplete lesions showed significant recovery (Table 4). Of the 19 incomplete patients, 17 recovered to near normal function (including three cases with initial sacral sparing only). Only two cases stayed at level D.

There was only one case showing significant neurological deterioration following manipulation and reduction. The patient was a 31 year old male who was attacked by a cow causing $\mathrm{C} 5 / 6$ bifacet dislocation. The reduction was achieved at $19 \mathrm{~h}$ post the injury. However, the deterioration was not immediately post manipulation but peaked at 3 days. The patient regained full function above the level of his complete cord lesion (Table 5). American Spinal Injury Association (ASIA) Scoring System was used to describe his sensory and motor function changes.

Other complications included respiratory compromise (three cases), requiring ICU care with or without artificial ventilation, DVT and pulmonary emboli which occurred in four cases. Among the three patients with respiratory compromise, one was due to ascending levels involving the diaphragm (same case shown in Table 5).

\section{Discussion}

The most important finding in our study is the correlation between co-existing fractures with dislocations and their inability to be reduced manually. The most common pattern of fracture is lateral mass fracture and/or facet joint fracture. Lateral mass fractures have been recognized as important due to their difficulty of detection and their major influence on stability. ${ }^{11}$ Even under these circumstances, manipulation under general anaesthesia will improve alignment of the displacement and offers considerable decom-

Table 1 Diagnostic classification

\begin{tabular}{lccc}
\hline & $\begin{array}{c}\text { Bifacet } \\
\text { dislocation }\end{array}$ & $\begin{array}{c}\text { Unifacet } \\
\text { dislocation }\end{array}$ & $\begin{array}{c}\text { Dislocation with } \\
\text { fractures }\end{array}$ \\
\hline $\begin{array}{l}\text { Number of cases } \\
\text { MUGA }\end{array}$ & 10 & 10 & 11 \\
$\begin{array}{l}\text { Reduced } \\
\text { Not reduced }\end{array}$ & 9 & 8 & 2 \\
& 1 & $\begin{array}{c}\text { One by } \\
\text { traction alone } \\
\text { One delayed } \\
\text { admission }\end{array}$ & $\begin{array}{c}2 \text { cases need } \\
\text { primary } \\
\text { ORIF }\end{array}$ \\
& \\
\end{tabular}

Table 2 Internal fixations

\begin{tabular}{lccc}
\hline & $\begin{array}{c}\text { Bifacet } \\
\text { dislocation }\end{array}$ & $\begin{array}{c}\text { Unifacet } \\
\text { dislocation }\end{array}$ & $\begin{array}{c}\text { Fracture } \\
\text { dislocations }\end{array}$ \\
\hline 'H' Plate & 9 & 8 & 4 \\
Posterior wiring & 1 & 2 & 7 \\
& & One due to \\
& delayed admission \\
& and one \\
& re-dislocation \\
\hline
\end{tabular}

Table 3 Neurological impairment

\begin{tabular}{lccc}
\hline & $\begin{array}{c}\text { Bifacet } \\
\text { dislocation }\end{array}$ & $\begin{array}{c}\text { Unifacet } \\
\text { dislocation }\end{array}$ & $\begin{array}{c}\text { Fracture } \\
\text { dislocations }\end{array}$ \\
\hline Complete & 5 & 3 & 5 \\
Incomplete & 3 & 6 & 4 \\
Minimal & 1 & 1 & 2 \\
None & 1 & 3 & \\
\hline
\end{tabular}

Table 4 Frankel scale outcome

\begin{tabular}{|c|c|c|c|c|c|c|}
\hline & & $A$ & $B$ & $C$ & $D$ & $E$ \\
\hline \multirow[t]{2}{*}{ Bifacet Group } & Admission & 5 & \multirow{2}{*}{\multicolumn{4}{|c|}{$2------2-=-5$}} \\
\hline & Discharge & 5 & & & & \\
\hline \multirow[t]{2}{*}{ Unifacet } & Admission & 0 & & & \multirow{2}{*}{\multicolumn{2}{|c|}{$9--10$}} \\
\hline & Discharge & & & & & \\
\hline \multirow[t]{2}{*}{ Fracture dislocation } & Admission & 5 & \multirow{2}{*}{\multicolumn{4}{|c|}{$1-\cdots-\cdots$}} \\
\hline & Discharge & 5 & & & 2 & \\
\hline
\end{tabular}


Table 5 Neurological deterioration

\begin{tabular}{lccc}
\hline & Motor & Sensory & Level \\
\hline Original scores & 5 & 14 & $\mathrm{C}_{5}$ \\
24 h post MUGA & 4 & 12 & $\mathrm{C}_{4}$ \\
3 days post MUGA & 0 & 12 & $\mathrm{C}_{4}$ \\
3 months & 10 & 16 & $\mathrm{C}_{5}$ \\
6 months & 13 & 16 & $\mathrm{C}_{5}$ \\
\hline
\end{tabular}

MRI: Mild cord compression; Extensive cord edema

pression effect. Our recommendation is to attempt to identify associated fractures prior to attempted reduction by careful study of the plain films supplemented by CT Scan if there is any doubt. Fracture of the vertebral body, on the other hand, would generally be obvious in plain films.

It is our philosophy not to force any reduction. If a dislocation cannot be reduced, more investigations are indicated or alternatively, an open reduction should be performed once the pattern of injury has been identified. By following these principles, we have observed very few neurological complications which if occurred, could be extremely serious. Our series also did not show the high mortality rate noted by Lee et $a l^{5}$ in the manipulation group. We feel this is due to skilled but gentle reduction, good ancillary care and attention to preventing anoxia with respiratory support if required.

The only case with clear post reduction deterioration was described in the section discussing results. The post reduction MRI scan in this patient identified no significant external contributing factors such as bony impingement, disc compression or haematoma. Spinal cord edema was regarded as the cause of his temporary ascending neurological picture. He eventually regained all functions generally expected for the same level of complete lesion (Table 5). Cord edema, or other intrinsic factors have been recognized as the most common mechanism of post reduction neurological deterioration. ${ }^{2,6}$ Post reduction CT or MRI of other patients without deterioration have show disc injuries but none were significant. In one patient who had a failed reduction, MRI imaging demonstrated bony and disc material behind the dislocated vertebral body, indicating potential risk if reduction had been attempted more vigorously. This particular patient was successfully reduced via a posterior approach followed by decompression anteriorly and ' $\mathrm{H}$ ' plate fixation. He returned to full time university in a year with near normal mobility and hand function, though he presented initially as a near complete tetraplegic with Frankel Scale B.

The rationale behind this approach (reduction first rather than decompression first) was due to the fact that the dislocated facet belongs also to the free lateral mass segment (with ipsilateral fractures of the lamina and pedicle) that was clearly irreducible by closed methods. Open reduction of the facet itself did not endanger the spinal cord as minimal movement would have occurred at the disc level. However, without such reduction, the relevant subluxed vertebral body would not be reduced even after disc decompression.

In our experience, the necessity of decompressing the disc prior to reduction recommended by some authors ${ }^{1,4}$ was not observed. Such an approach would also present technical difficulty as the destabilized spine would need a much stronger posterior fixation or three operations (Front-Back-Front) to finally stabilise the spine by anterior plating.

In our experience, manipulation under general anaesthesia is a safe and quick method to reduce cervical dislocations. Previous studies showed that prompt reduction of dislocation is of paramount importance for patients with significant neurological involvement. $^{1,5,9}$ Others, on the other hand, have suggested that patients with incomplete neurological damage can still make significant neurological recovery even when dislocations have been left unreduced. ${ }^{13}$ The timing of reduction in patients with minimal neurological deficits is less critical, though there have been reports of progressive paralysis after bilateral facet dislocation; most occurred outside spinal injury units. Up to now, little evidence is available to prove that delayed reduction will cause significant deterioration of the neurological impairment. Therefore, the absolute value of urgent reduction is still not clear despite the fact that it is practised almost routinely all over the world. Further controlled studies are needed to clarify the necessity of reduction and the timing of reduction.

The choice of reduction procedure is among manipulation under anaesthesia (MUA), traction with increasing weight and analgesia and open reduction. ${ }^{1,5,7}$ The MUA has the advantages of being the most rapid method of reduction, avoiding repeat radiation exposure and frequent monitoring required in rapid traction. This may be particularly true in experienced hands. However, one disadvantage of manipulation under general anaesthesia is the inability of the clinician to monitor the patient neurologically during the procedure. Therefore, such a procedure is not recommended for clinicians not experienced in spinal and spinal cord injuries and the manipulation procedure. With the recognition that anterior ' $\mathrm{H}$ ' plate fixation has become one of the best ways of achieving cervical stability, ${ }^{12}$ MUA also provides best pre-operative conditions and timing for this procedure. This will avoid posterior open reduction and wiring fixation which is mechanically less desirable and carries more post operative complications including prolonged rehabilitation and undesirable neck postures secondary to surgical damage to the posterior musculature.

\section{Acknowledgements}

I would like to express our sincere appreciation to Helen Fisher and Liz Jones for the preparation of the manuscript. We would also like to express our thanks to Dr Douglas Brown, Austin Hospital, Melbourne, Australia for proof reading the manuscript before publication. 


\section{References}

1 Rizzolo SJ et al. Cervical spine trauma [Review]. Spine 1994; 19: $2288-2298$.

2 Mahale YJ et al. Neurological complications of the reduction of cervical spine dislocations. JBJS-Br V 1993; 75: $403-409$.

3 Roberston PA, Ryan MD. Neurological deterioration after reduction of cervical subluxation. Mechanical compression of disc tissues. $J B J S-B r V$ 1992; 74: $224-227$.

4 Olernd C. Compression of the cervical spinal cord after reduction of fracture dislocations. Report of two cases. Acta Orth Scand 1991; 62: 599-601.

5 Lee AS et al. Rapid traction for reduction of cervical spine dislocations. JBJS-Br V 1994; 76: 352 - 356 .

6 Mahale YJ. Progessive paralysis after bilateral facet dislocation of the cervical spinal. $J B J S-B r V$ 1992; 74: $219-223$.

7 Beyer CA. Unilateral facet dislocations and fracture - dislocations of the cervical spine: a review. Orthopaedics 1992; 15: 311 315.

8 Hadley MN et al. Facet fracture - dislocation injuries of the cervical spine. Neurosurgery 1992; 30: 661-666.

9 Wolf A et al. Operative management of bilateral facet dislocation. J of Neurosurgery 1991; 75: 883-890.

10 Rizzolo SJ et al. Intervertebral disc injury complicating cervical spinal trauma. Spine 1991; 16: 187-189.

11 Xiong X, Bean A. Lateral mass fracture and its instability in the cervical spine and spinal cord injuries. Presented 1995 Annual meeting of IMSOP, Sydney, Australia.

12 Inglis G. Orthopaedic Surgeon, personal communication.

13 Frankel HL et al. The value of postural reduction in the initial management of closed injuries of the spine with paraplegia and tetraplegia. Paraplegia 1969; 7: 179-192. 